Huixing $\mathrm{Li}^{\star}$, Xinlong Han and Bin Cao

\title{
Crystal structure of catena-poly[trans-tetraaqua $\left(\mu_{2}-1,1^{\prime}\right.$-(biphenyl-4,4'-diyl)bis( $1 \mathrm{H}$-imidazol)- $\left.k^{2} \mathrm{~N}: \mathrm{N}^{\prime}\right)$ cobalt(II)] dinitrate $-1,1^{\prime}$-(biphenyl-4, 4'-diyl)bis(1H-imidazol) - water (1/3/2), $\mathrm{C}_{72} \mathrm{H}_{68} \mathrm{CoN}_{18} \mathrm{O}_{12}$
}
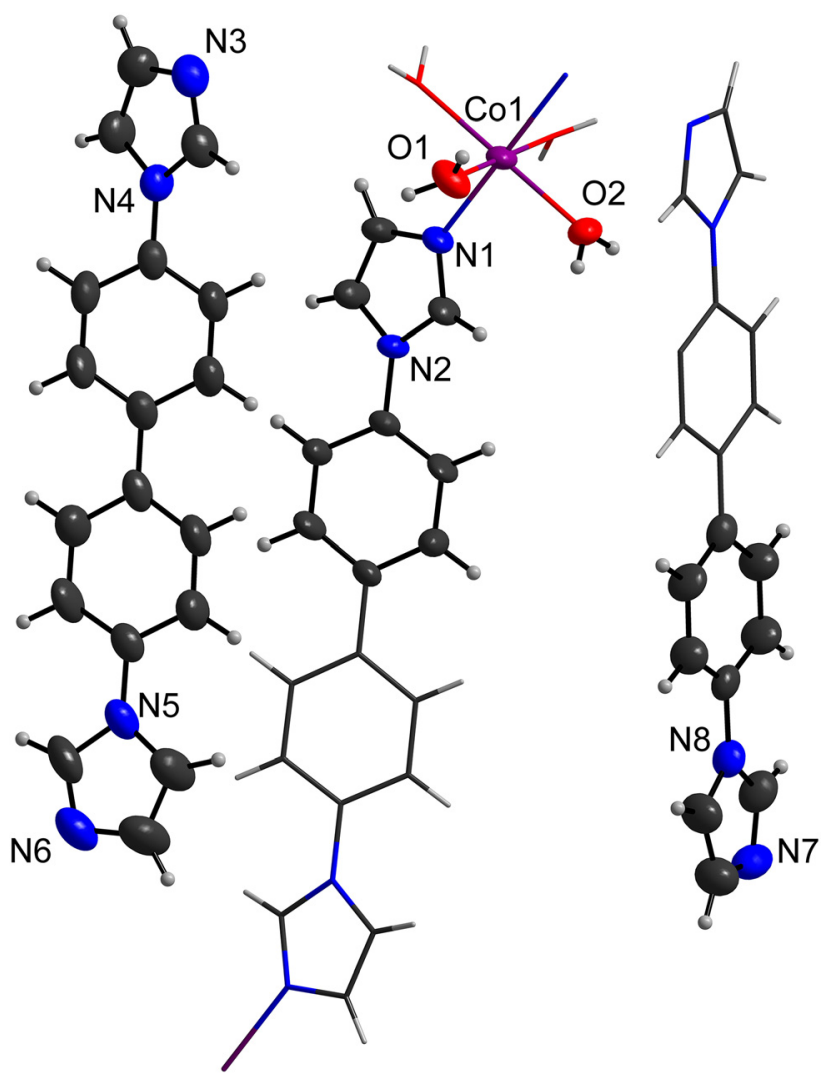

https://doi.org/10.1515/ncrs-2020-0572

Received November 4, 2020; accepted December 18, 2020; published online January 13, 2021

*Corresponding author: Huixing Li, Henan Key Laboratory of Industrial Microbial Resources and Fermentation Technology, Nanyang Institute of Technology, 473004, Nanyang, Henan, People's Republic of China; and School of Biological and Chemical Engineering, Nanyang Institute of Technology, 473004, Nanyang, Henan, People's Republic of China, E-mail: lucky2002566@163.com. https://orcid. org/0000-0002-1340-6179

Xinlong Han and Bin Cao, Henan Key Laboratory of Industrial Microbial Resources and Fermentation Technology, Nanyang Institute of Technology, 473004, Nanyang, Henan, People's Republic of China; and School of Biological and Chemical Engineering, Nanyang Institute of Technology, 473004, Nanyang, Henan, People’s Republic of China
Abstract

$\mathrm{C}_{72} \mathrm{H}_{68} \mathrm{CoN}_{18} \mathrm{O}_{12}$, triclinic, $P \overline{1}$ (no. 2), $a=9.2801(10) \AA$, $b=11.6017(11) \AA, c=16.4968(15) \AA, \alpha=96.294(8)^{\circ}$, $\beta=96.371(8)^{\circ}, \gamma=105.025(9)^{\circ}, V=1687.0(3) \AA^{3}, Z=1$, $R_{g t}(F)=0.0631, w R_{\text {ref }}\left(F^{2}\right)=0.1043, T=293(2) \mathrm{K}$.

CCDC no.: 2051182

Table 1 contains crystallographic data and Table 2 contains the list of the atoms including atomic coordinates and displacement parameters.

Table 1: Data collection and handling.

$\begin{array}{ll}\text { Crystal: } & \text { Purple block } \\ \text { Size: } & 0.25 \times 0.23 \times 0.20 \mathrm{~mm} \\ \text { Wavelength: } & \text { Mo } K \alpha \text { radiation }(0.71073 \AA) \\ \mu: & 0.33 \mathrm{~mm}^{-1} \\ \text { Diffractometer, scan mode: } & \text { Bruker APEX-II, } \varphi \text { and } \omega \\ \theta_{\text {max }}, \text { completeness: } & 25.0^{\circ},>99 \% \\ N(h k l)_{\text {measured }}, N(h k l)_{\text {unique }}, R_{\text {int }}: & 11546,5939,0.081 \\ \text { Criterion for } I_{\text {obs }}, N(h k l)_{\text {gt }}: & I_{\text {obs }}>2 \sigma\left(I_{\text {obs }}\right), 3359 \\ N(\text { param })_{\text {refined }}: & 505 \\ \text { Programs: } & \text { Bruker [1], SHELX [2] }\end{array}$

\section{Source of material}

All chemicals and solvents were of analytical grade and used without further purification. The title compound was prepared by the hydrothermal method. A mixture of $\mathrm{Co}\left(\mathrm{NO}_{3}\right)_{2} \cdot 6 \mathrm{H}_{2} \mathrm{O}$ (1 mmol, $\left.0.291 \mathrm{~g}\right), 1,1^{\prime}$-(biphenyl4,4'-diyl)bis(1H-imidazol) (bibp) (1 mmol, $0.288 \mathrm{~g})$, dimethylformamide $(6 \mathrm{ml})$ and $\mathrm{H}_{2} \mathrm{O}(2 \mathrm{ml})$ were placed in a $25 \mathrm{~mL}$ Teflon-lined autoclave at $413 \mathrm{~K}$ for $72 \mathrm{~h}$, then cooled to room temperature. Purple block crystals were obtained. 
Table 2: Fractional atomic coordinates and isotropic or equivalent isotropic displacement parameters $\left(\AA^{2}\right)$.

\begin{tabular}{|c|c|c|c|c|}
\hline Atom & $x$ & $y$ & $z$ & $U_{\text {iso }} * / U_{\text {eq }}$ \\
\hline Co1 & 1.000000 & 1.000000 & 0.500000 & $0.0353(2)$ \\
\hline 01 & $1.0038(3)$ & $0.8289(2)$ & $0.44874(16)$ & $0.0541(8)$ \\
\hline $\mathrm{H} 1 \mathrm{~A}$ & 1.0705 (19) & $0.7956(18)$ & $0.466(2)$ & 0.081 * \\
\hline $\mathrm{H} 1 \mathrm{~B}$ & $0.9293(18)$ & $0.7712(14)$ & 0.4250 (19) & $0.081^{*}$ \\
\hline 02 & $1.1532(3)$ & $1.0772(2)$ & 0.42165 (13) & $0.0459(7)$ \\
\hline $\mathrm{H} 2 \mathrm{~A}$ & $1.158(4)$ & 1.0397 (18) & $0.3752(8)$ & 0.069 * \\
\hline $\mathrm{H} 2 \mathrm{~B}$ & $1.163(4)$ & $1.1499(7)$ & $0.4138(14)$ & $0.069^{*}$ \\
\hline N1 & $0.8093(3)$ & $0.9885(2)$ & $0.41180(16)$ & $0.0358(7)$ \\
\hline N2 & $0.6616(3)$ & $0.9836(2)$ & $0.29555(16)$ & $0.0350(7)$ \\
\hline N3 & $0.7781(4)$ & $0.2720(3)$ & $0.4883(2)$ & $0.0570(10)$ \\
\hline N4 & $0.6408(4)$ & $0.2913(3)$ & $0.3748(2)$ & 0.0481 (9) \\
\hline N5 & $0.3760(4)$ & $0.3408(3)$ & $-0.2191(2)$ & $0.0506(9)$ \\
\hline N6 & $0.2387(4)$ & $0.3217(3)$ & $-0.3417(2)$ & $0.0698(12)$ \\
\hline N7 & $0.1667(4)$ & $0.3067(3)$ & $0.3836(2)$ & $0.0612(10)$ \\
\hline N8 & $0.1175(3)$ & $0.4093(3)$ & 0.28392 (19) & $0.0450(8)$ \\
\hline $\mathrm{C} 1$ & $0.8038(4)$ & $0.9973(3)$ & $0.3322(2)$ & $0.0389(10)$ \\
\hline $\mathrm{H} 1$ & 0.887689 & 1.011180 & 0.304849 & $0.047^{*}$ \\
\hline $\mathrm{C} 2$ & $0.6624(4)$ & $0.9681(3)$ & $0.4258(2)$ & $0.0450(10)$ \\
\hline $\mathrm{H} 2$ & 0.630182 & 0.957500 & 0.476524 & $0.054^{*}$ \\
\hline $\mathrm{C3}$ & $0.5711(4)$ & $0.9656(3)$ & $0.3555(2)$ & $0.0497(11)$ \\
\hline H3 & 0.467304 & 0.954003 & 0.349193 & 0.060 * \\
\hline $\mathrm{C} 4$ & $0.6161(4)$ & $0.9879(3)$ & $0.2102(2)$ & $0.0334(9)$ \\
\hline $\mathrm{C} 5$ & $0.4714(4)$ & $0.9269(3)$ & $0.1737(2)$ & $0.0441(10)$ \\
\hline H5 & 0.404050 & 0.882063 & 0.203798 & $0.053^{*}$ \\
\hline C6 & $0.4277(4)$ & $0.9330(3)$ & $0.0921(2)$ & $0.0478(11)$ \\
\hline H6 & 0.329225 & 0.893042 & 0.068277 & $0.057^{*}$ \\
\hline $\mathrm{C7}$ & $0.5244(4)$ & $0.9962(3)$ & $0.04438(18)$ & $0.0348(9)$ \\
\hline $\mathrm{C} 8$ & $0.6685(4)$ & $1.0564(3)$ & $0.0828(2)$ & $0.0564(12)$ \\
\hline H8 & 0.736570 & 1.100478 & 0.052723 & $0.068^{*}$ \\
\hline C9 & $0.7140(4)$ & $1.0527(3)$ & $0.1652(2)$ & $0.0542(12)$ \\
\hline H9 & 0.811462 & 1.094468 & 0.189772 & $0.065^{*}$ \\
\hline C10 & $0.6345(5)$ & $0.2678(4)$ & $0.5039(3)$ & $0.0612(12)$ \\
\hline $\mathrm{H} 10$ & 0.600940 & 0.258794 & 0.554485 & $0.073^{*}$ \\
\hline C11 & $0.5491(5)$ & $0.2788(4)$ & $0.4344(3)$ & $0.0590(12)$ \\
\hline H11 & 0.447978 & 0.277956 & 0.428453 & 0.071 * \\
\hline C12 & $0.7769(4)$ & $0.2861(3)$ & 0.4109 (3) & $0.0542(11)$ \\
\hline H12 & 0.860263 & 0.292013 & 0.383447 & $0.065^{\star}$ \\
\hline C13 & $0.6016(4)$ & $0.3016(3)$ & $0.2902(2)$ & $0.0433(10)$ \\
\hline C14 & $0.4634(4)$ & $0.2341(3)$ & $0.2476(3)$ & $0.0550(12)$ \\
\hline H14 & 0.395416 & 0.183114 & 0.274212 & $0.066^{*}$ \\
\hline C15 & $0.4269(4)$ & $0.2425(4)$ & $0.1661(3)$ & $0.0579(12)$ \\
\hline H15 & 0.332361 & 0.198385 & 0.138623 & $0.069^{*}$ \\
\hline C16 & $0.5262(4)$ & $0.3146(3)$ & $0.1234(2)$ & $0.0430(10)$ \\
\hline C17 & $0.6656(4)$ & $0.3813(3)$ & $0.1673(2)$ & $0.0539(11)$ \\
\hline H17 & 0.735348 & 0.430710 & 0.140784 & $0.065^{\star}$ \\
\hline C18 & $0.7010(4)$ & $0.3745(3)$ & $0.2497(2)$ & $0.0524(11)$ \\
\hline H18 & 0.793941 & 0.420220 & 0.278205 & $0.063^{*}$ \\
\hline C19 & $0.4886(4)$ & $0.3222(3)$ & $0.0343(3)$ & 0.0454 (10) \\
\hline $\mathrm{C} 20$ & $0.3460(5)$ & $0.2666(4)$ & $-0.0087(3)$ & $0.0613(12)$ \\
\hline $\mathrm{H} 20$ & 0.272345 & 0.224095 & 0.019095 & $0.074^{*}$ \\
\hline $\mathrm{C} 21$ & $0.3093(4)$ & $0.2721(4)$ & $-0.0913(3)$ & $0.0636(13)$ \\
\hline H21 & 0.212724 & 0.232601 & -0.118275 & $0.076^{*}$ \\
\hline $\mathrm{C} 22$ & $0.4141(4)$ & $0.3354(3)$ & $-0.1338(2)$ & $0.0461(10)$ \\
\hline $\mathrm{C} 23$ & $0.5564(4)$ & $0.3932(4)$ & $-0.0931(3)$ & $0.0607(12)$ \\
\hline $\mathrm{H} 23$ & 0.628751 & 0.438122 & -0.120528 & $0.073^{*}$ \\
\hline
\end{tabular}

Table 2: (continued)

\begin{tabular}{|c|c|c|c|c|}
\hline Atom & $x$ & $y$ & $z$ & $U_{\text {iso }} * / U_{\text {eq }}$ \\
\hline C24 & 0.5901 (4) & $0.3837(3)$ & $-0.0109(3)$ & $0.0581(12)$ \\
\hline $\mathrm{H} 24$ & 0.687728 & 0.421311 & 0.015320 & $0.070^{\star}$ \\
\hline $\mathrm{C} 25$ & $0.2369(5)$ & $0.3047(4)$ & $-0.2648(3)$ & 0.0675 (14) \\
\hline $\mathrm{H} 25$ & 0.149281 & 0.271190 & -0.243565 & $0.081^{*}$ \\
\hline $\mathrm{C} 26$ & $0.3871(6)$ & $0.3714(4)$ & $-0.3473(3)$ & 0.0792 (15) \\
\hline $\mathrm{H} 26$ & 0.424473 & 0.393081 & -0.395106 & $0.095^{\star}$ \\
\hline $\mathrm{C} 27$ & $0.4713(5)$ & $0.3843(4)$ & $-0.2733(3)$ & 0.0766 (15) \\
\hline $\mathrm{H} 27$ & 0.575213 & 0.416802 & -0.261092 & $0.092^{\star}$ \\
\hline $\mathrm{C} 28$ & $0.1692(5)$ & $0.4196(4)$ & $0.4179(3)$ & $0.0702(14)$ \\
\hline $\mathrm{H} 28$ & 0.188047 & 0.447873 & 0.474074 & $0.084^{*}$ \\
\hline C29 & $0.1398(5)$ & $0.4853(4)$ & $0.3572(3)$ & $0.0636(13)$ \\
\hline $\mathrm{H} 29$ & 0.135770 & 0.564945 & 0.363992 & $0.076^{*}$ \\
\hline C 30 & $0.1349(4)$ & $0.3041(4)$ & $0.3042(3)$ & $0.0558(12)$ \\
\hline $\mathrm{H} 30$ & 0.125348 & 0.236641 & 0.265719 & $0.067^{\star}$ \\
\hline C31 & $0.0848(4)$ & $0.4368(3)$ & $0.2028(2)$ & $0.0438(10)$ \\
\hline C32 & $0.0821(4)$ & $0.5500(3)$ & $0.1893(2)$ & 0.0515 (11) \\
\hline $\mathrm{H} 32$ & 0.103378 & 0.611364 & 0.233803 & $0.062^{*}$ \\
\hline C33 & 0.0486 (4) & $0.5747(3)$ & $0.1112(2)$ & $0.0512(11)$ \\
\hline H33 & 0.046078 & 0.652578 & 0.103957 & $0.061^{*}$ \\
\hline C34 & $0.0180(4)$ & $0.4864(3)$ & $0.0423(2)$ & $0.0418(10)$ \\
\hline C35 & 0.0209 (4) & $0.3720(3)$ & $0.0576(2)$ & 0.0537 (11) \\
\hline H35 & -0.000031 & 0.310292 & 0.013362 & $0.064^{\star}$ \\
\hline C36 & $0.0534(4)$ & $0.3462(3)$ & 0.1357 (3) & $0.0562(12)$ \\
\hline H36 & 0.054475 & 0.268310 & 0.143628 & $0.067^{\star}$ \\
\hline 03 & $0.0674(4)$ & $0.8692(4)$ & $0.1503(3)$ & $0.1113(15)$ \\
\hline 04 & 0.1134 & $1.0287(4)$ & $0.2370(3)$ & 0.1302 (19) \\
\hline $05 A^{a}$ & 0.0664 (13) & $1.0425(10)$ & $0.1122(7)$ & $0.113(4)$ \\
\hline $\mathrm{N} 9^{\mathrm{a}}$ & $0.0805(10)$ & 0.9815 (12) & 0.1664 (9) & $0.083(3)$ \\
\hline $01 W A^{a}$ & $0.1943(11)$ & $0.8175(8)$ & $0.3102(6)$ & 0.107 (3) \\
\hline$H 1 W A^{a}$ & 0.146102 & 0.839748 & 0.347110 & 0.161 * \\
\hline$H 1 W B^{a}$ & 0.150363 & 0.821427 & 0.262940 & $0.161^{*}$ \\
\hline $05 B^{a}$ & 0.1640 (15) & $0.8836(13)$ & $0.2780(8)$ & $0.161(6)$ \\
\hline $\mathrm{N} \mathrm{B}^{\mathrm{a}}$ & 0.1127 (11) & 0.9224 (11) & $0.2226(8)$ & $0.086(3)$ \\
\hline $01 W^{a}$ & 0.0484 (14) & $1.0747(9)$ & $0.0584(6)$ & 0.118 (4) \\
\hline$H 1 W C^{a}$ & 0.003260 & 1.046526 & 0.009772 & $0.177^{\star}$ \\
\hline$H 1 W D^{a}$ & 0.111260 & 1.035676 & 0.071562 & $0.177^{*}$ \\
\hline
\end{tabular}

a Occupancy: 0.5

\section{Experimental details}

$\mathrm{H}$ atoms bound to $\mathrm{C}$ atoms were placed in calculated positions and refined as riding on their parent atoms, with C$\mathrm{H}=0.93 \AA$ and with $U_{\text {iso }}(\mathrm{H})=1.2 U_{\text {eq }}(\mathrm{C})$. The O-bound $\mathrm{H}$ atoms were located in a difference Fourier map and refined with a distance restraint of $\mathrm{O}-\mathrm{H}=0.85 \AA$, and with $U_{\text {iso }}(\mathrm{H})$ set to 1.5 $U_{\text {eq }}(0)$. The uncoordinated water molecules and the nitrate anions are disordered over two postions in this structure.

\section{Comment}

Imidazole is a versatile $\mathrm{N}$-donor organic ligand in construction of coordination polymers because of the potential 
coordination sites [3-5]. As a imidazole derivative, 1,1'(biphenyl-4,4'-diyl)bis(1H-imidazol) (bibp) was widely used to synthesise coordination polymers due to different coordination modes of the bibp ligand [6-11].

The asymmetric unit of the crystal structure consists of half of a Co(II) center located on a crystallographic inversion center, one half of a bibp ligand, two coordinated water molecules, one and a half free bibp ligands (see the Figure). The nitrate counter anion and one more uncoordinated water molecule are both disordered and not shown in the Figure. The Co(II) ion is six-coordinated by four $\mathrm{O}$ atoms from two pairs of symmetry related water molecules $(\mathrm{Co}-\mathrm{O}=2.081(2)-$ $2.120(2) \AA$ ), and two $\mathrm{N}$ atoms from two symmetry related bibp ligands $(\mathrm{Co}-\mathrm{N}=2.129(3) \AA$ ), forming a distorted octahedral geometry. The coordinated bibp ligands link adjacent $\mathrm{Co}(\mathrm{II})$ ions into a polymeric chain structure with the Co-Co distance of $18.008 \AA$ along the $c$ axis. In the crystal structure, $\mathrm{O}-\mathrm{H} \cdots \mathrm{O}$ and $\mathrm{O}-\mathrm{H} \cdots \mathrm{N}$ hydrogen bonds link molecules and ions into a threedimensional supermolecular framework.

Author contribution: All the authors have accepted responsibility for the entire content of this submitted manuscript and approved submission.

Research funding: Natural Science Foundation of Henan Province of China (182300410151), Scientific and Technological Project of Henan Province (162102210114), Key Scientific Research Project of College and University in Henan Province (17A610003) and Henan Key Laboratory of Industrial Microbial Resources and Fermentation Technology Open Project (HIMFT20200206).

Conflict of interest statement: The authors declare no conflicts of interest regarding this article.

\section{References}

1. Bruker. APEX2, SAINT and SADABS; Bruker AXS Inc.: Madison, Wisconsin, USA, 2008.

2. Sheldrick G. M. A short history of SHELX. Acta Crystallogr. 2008, A64, 112-122.

3. Sun Y., Chen Y., Wang D. Crystal structure of poly[bis $\left(\mu_{3}-(1-(3,5-\right.$ di(1H-imidazol-1-yl)phenyl)-1H-imidazole- $\left.\kappa^{3} N: N^{\prime}: N^{\prime \prime}\right)$ cobalt(II)] dinitrate - $N, N$ - dimethylformamide (1/4), $\mathrm{C}_{42} \mathrm{H}_{52} \mathrm{~N}_{18} \mathrm{O}_{10} \mathrm{Co}$. Z. Kristallogr. NCS 2020, 235, 1303-1305.

4. Li T., Xing Z. Crystal structure of hexa- $\mu_{2}$-chlorido- $\mu_{4^{-}}$ oxidotetrakis (1-vinyl-1H-imidazole- $K N$ ) tetracopper(II), $\mathrm{C}_{20} \mathrm{H}_{24} \mathrm{Cu}_{4} \mathrm{Cl}_{6} \mathrm{~N}_{8}$ O. Z. Kristallogr. NCS 2019, 234, 363-365.

5. Çetinkaya F., Kürkçüoğlu G. S., Yeşilel O. Z., Hökelek T., Dal H. One-dimensional cyano-bridged heterometallic $(\mathrm{Cu} / \mathrm{Ni}$ and $\mathrm{Cu} / \mathrm{Pd})$ complexes with 1-ethylimidazole. Polyhedron 2012, 47, 126-133.

6. Hu Q., Zheng Q.-M., Ma X.-R., Lai Z.-Z., Ye T.-Q., Qin L. One luminescence probe and the impact of dye-adsorption on the luminescent property. Polyhedron 2020, 177, 114323.

7. Voda I., Makhloufi G., Druta V., Lozan V., Shova S., Bourosh P., Kravtsov V., Janiak C. Mixed-ligand coordination compounds based on the rigid 4,4'-bis(1-imidazolyl)biphenyl and pyridinedicarboxylate ligands. Inorg. Chim. Acta. 2018, 482, 526-534.

8. Li Z.-X., Gou X.-F., Jie W., Zha G., Wang T., Xu Y. One pillared- layer $\alpha$-Po framework with a rare tetracobalt-formate $(4,4)$ sheet exhibiting a field-induced magnetic transition. Inorg. Chem. Commun. 2014, 41, 58-61.

9. Zhang W., Hao L. Coordination polymers $\left(\mathrm{Ni}^{\prime \prime}\right.$ and $\left.\mathrm{Co}^{\prime \prime}\right)$ based on the rigid bridging ligand, 4,4'-bis(imidazol-1-yl)biphenyl, with $\left(4^{6}\right)$ topology. J. Coord. Chem. 2013, 66, 2100-2117.

10. Zhang C.-L., Qin L., Zheng H.-G. Synthesis, characterization and crystal structure of one Co(II) complex with 4,4'-bisimidazolylbiphenyl and $m$-phthalic acid. Inorg. Chem. Commun. 2013, 36, 192-194.

11. Li Z.-X., Zhao J.-P., Sañudo E. C., Ma H., Pan Z.-D., Zeng Y.-F., Bu X.-H. New 3D coordination polymers constructed from pillared metal- formate Kagomlayers exhibiting spin canting only in the nickel(II) complex. Inorg. Chem. 2009, 48, 11601-11607. 